2017

\title{
The Forces Affecting a Sailboat
}

Kelly Stukbauer

University of South Florida

Advisors:

David Milligan, Mathematics and Statistics

Darío Arena, Physics

Problem Suggested By: Kelly Stukbauer

Follow this and additional works at: https://digitalcommons.usf.edu/ujmm

Part of the Mathematics Commons

UJMM is an open access journal, free to authors and readers, and relies on your support: Donate Now

\section{Recommended Citation}

Stukbauer, Kelly (2017) "The Forces Affecting a Sailboat," Undergraduate Journal of Mathematical Modeling: One + Two: Vol. 8: Iss. 1, Article 2.

DOI: http://doi.org/10.5038/2326-3652.8.1.4883

Available at: https://digitalcommons.usf.edu/ujmm/vol8/iss1/2 


\title{
The Forces Affecting a Sailboat
}

\begin{abstract}
The objective of this project is to determine the specific effects of the various forces acting on a sailboat. Through this project, a greater understanding of both the physics behind sailing, and the calculus behind that physics, shall be gained.
\end{abstract}

Keywords

sailboat, pressure, drag, fluid dynamics

Creative Commons License

(c) (i) (8)

This work is licensed under a Creative Commons Attribution-Noncommercial-Share Alike 4.0 License. 


\section{PROBLEM STATEMENT}

The net force acting on a sailboat can be obtained by examining each component force separately and then combining them. This project implements vectors in three dimensions and vector addition as well as the concepts of buoyancy, differences in pressure, drag, and fluid dynamics to evaluate the forces on a sailboat. It is important to note that although the results obtained from this project are based on the collegiate FJ sailboat, they can be applied to any sailboat. The collegiate FJ was chosen because it is commonly sailed by college sailing teams, including the University of South Florida's sailing team.

\section{MOTIVATION}

Although sailboats have been in existence for thousands of years and lateen sails, the triangular shaped sails that power most modern sailboats, have been in use since medieval times, there is still much to be learned from the interaction of the various forces that allow the sailboat to move forward (Encyclopædia Britannica, 2015). Through the understanding of these forces, one not only gains deeper insight into how the forces of the natural world (more specifically the wind) can be harnessed, but also how different physical forces interact in a system. The interaction of these forces can be evaluated using three-dimensional vectors and trigonometric functions learned in Calculus III. In this investigation, the Cartesian coordinate system is used rather than a polar or parametric coordinate system because Cartesian coordinates are more conducive to the computation of the equations in this investigation. Through the implementation of these concepts, the various forces affecting a sailboat can be broken into components, and these components can be combined to obtain the components of the net force. Once the components 
of the net force are obtained, the net force can be evaluated. This evaluation allows the true motion of the sailboat to be understood and analyzed.

This research is beneficial from both an engineering and scientific standpoint. It is beneficial from an engineering standpoint in that it broadens the understanding of a complex system and provides a method for breaking down intricate systems into components that can be evaluated separately and then later combined to obtain the net effect, which can be used to design more efficient systems as well as make existing systems operate more efficiently. From the scientific standpoint, it is beneficial because it creates an increased awareness of the physical world and how the laws of physics interact to produce the motion of a sailboat. This research can be directly applied to improving the design of sailboats, as well as improving the performance of existing sailboats.

\section{MATHEMATICAL DESCRIPTION AND SOLUTION APPROACH}

For consistency, the axes will be defined as follows. The positive $y$-direction is towards the starboard side of the boat (Figure 1), the positive $x$-direction is towards the stern (Figure 1), and the positive z-direction is upwards. See the appendix of nautical terms.

It is important to note that, when boat-specific values are needed for calculations, the numbers are based upon the collegiate FJ specifications. The collegiate FJ is a commonly sailed two person dingy that is similar to many other two person sailboats on the market. Thus, although 


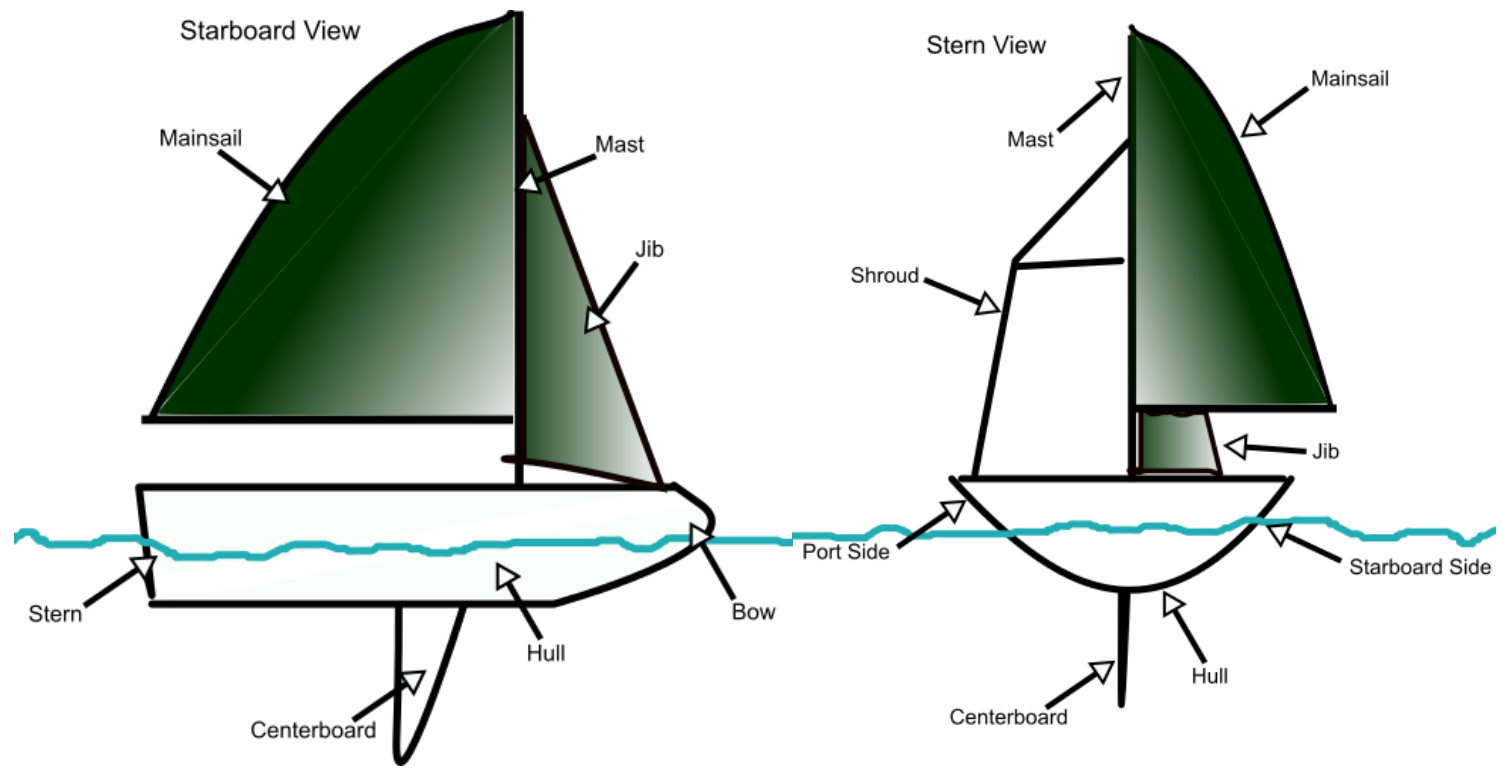

Figure 1: The parts of a sailboat

these calculations are based upon the collegiate FJ, they can be easily applied to any sailboat following some simple substitutions.

The calculation of the force due to gravity caused by the combined weight of the sailboat and sailors $\left(F_{m g}\right)$ is given by the following

$$
F_{m g}=M g=(-9.8) M
$$

where $M$ is the combined mass of sailboat and sailors and $g$ is the acceleration due to gravity $\left(-9.8 \mathrm{~m} / \mathrm{s}^{2}\right)$. The force (1) acts downward in the negative z-direction. 
The buoyant force $\left(F_{b}\right)$ acts in the positive z-direction. This makes sense because the sailboat is floating and the buoyant force always acts in the opposite direction of $F_{m g}$. Therefore, $F_{m g}$ must be both equal and opposite the buoyant force.

$$
-F_{m g}=F_{b}
$$

Using formula (2), the volume $V$ of water displaced by the boat can be calculated. With

$$
\begin{gathered}
\rho_{\text {water }}=\text { density of water } \\
V=\text { volume of water displaced by boat } \\
F_{b}=\rho_{\text {water }} g V \\
V=\frac{F_{b}}{\rho_{\text {water }} g}
\end{gathered}
$$

and substituting $-F_{m g}$ for $F_{b}$ yields

$$
V=\frac{-F_{m g}}{\rho_{\text {water }} g} .
$$

At this point it is important to note that since $F_{m g}$ and $F_{b}$ are equal and opposite, they cancel each other out. This is logical because as the sailboat travels through the water it is neither moving upwards nor downwards (since it is assumed to be neither foiling nor sinking). Therefore, the net force in the z-direction is zero, does not affect the motion of the boat, and will not be discussed further.

For this investigation the force caused by the difference in high and low pressures created by the sails is referred to as the force of the wind, $F_{w}$. This stems from the fact that this force results from the interactions between the sails and the wind. Specifically, the force can be explained by 
Bernoulli's principle because the air particles on the leeward side (in this case the starboard side) of the sail are forced to move faster than the air particles on the windward side (in this case the port side) of the sail since they must cover more distance. Using Bernoulli's equation, as the velocity increases the pressure decreases and as the velocity decreases the pressure increases. Bernoulli's equation is used to relate this difference in velocity between the two sides of the sail and this difference in pressure between the two sides of the sail. This difference in pressure (caused by the difference in velocity) creates a force that can be denoted by the formula

$$
F_{w}=\frac{\Delta P M}{\rho_{\text {air }} d}
$$

where $\Delta P$ is the change in pressure, $M$ is the combined mass of the sailboat and the sailors, $\rho_{\text {air }}$ is the density of air, and $d$ is the distance between the two pressures (Verner E. Suomi Virtual Museum, 1999). In this case, the distance is small because the pressure difference occurs on the opposite sides of the sail, which does not cover much distance. The resultant force is assumed to be at an angle of approximately $45^{\circ}$ toward starboard from the bow of the boat. The force is assumed to be towards starboard because the wind is coming over the port side of the boat, causing the high pressure region to be on the port side and the low pressure to be on the starboard side. Therefore, the $x$-component is in the negative $x$-direction and denoted by

$$
F_{w x}=-\frac{1}{\sqrt{2}} F_{w}=-\frac{1}{\sqrt{2}} \frac{\Delta P M}{\rho_{a i r} d}
$$

and the $y$-component can be denoted by

$$
F_{w y}=\frac{1}{\sqrt{2}} F_{w}=\frac{1}{\sqrt{2}} \frac{\Delta P M}{\rho_{\text {air }} d} .
$$


The resistance force, denoted $F_{r}$ acts in the positive $x$-direction and acts counter to the force $F_{w x}$ that acts in the negative $x$-direction. This force can be related to the force of friction in that it is a resistive force but its characteristics are different from the typical frictional forces because it deals with fluids and it is dependent upon velocity in a non-trivial way. At low speeds, the water flowing along the hull of the boat exhibits laminar flow. In these cases, the resistance is very small, but normally sailboats operate at speeds greater than those required to achieve laminar flow. Therefore, for this investigation, the sailboat's speed will be assumed to exceed the speed needed for laminar flow. This higher speed results in the water experiencing turbulent flow instead of laminar flow, meaning that the motion of the water as it passes the hull of the sailboat is more chaotic. Thus, when calculating the resistance, Reynolds number $(R)$ must first be obtained using the formula

$$
R=\frac{L v}{\frac{\mu}{\rho}}
$$

where $v$ is the velocity, $\rho$ is the density of the water, $\mu$ is the viscosity of the water, and $L$ is the length of the boat. The coefficient of friction $\left(\mu_{R}\right)$ can be calculated using

$$
\mu_{R}=\frac{64}{R}
$$

where $R$ is Reynolds number (LMNO Engineering, 2008). Using the $\mu_{R}$ obtained from formula (5), one can then calculate $F_{r}$ using the formula

$$
F_{r}=\mu_{R} A v^{2}
$$


where $A$ is the surface area of the hull that is underwater and $v$ is the velocity at which the sailboat is moving through the water.

The sailboat is prevented from excessively slipping sideways by the centerboard. The centerboard protrudes from the hull of the sailboat. It appears to be very narrow when viewed from the bow or stern of the boat such that it adds little resistance to the sailboat's forward motion. However, when viewed from the starboard or port side of the boat, the centerboard appears to be a relatively large plane. It is this plane that provides the resistance that acts in the negative $y$-direction and partially counters the $F_{w y}$. This reduces the amount that the sailboat slips sideways. This force, denoted $F_{c b w}$ because it is caused by the interaction of the centerboard and the water, can be calculated by

$$
F_{c b w}=-\frac{C \rho A_{c b} v^{2}}{2}
$$

where $C$ is the drag coefficient, $\rho$ is the density of the water, $A_{c b}$ is the area of the centerboard, and $v$ is the velocity. The drag coefficient, $C$, can be calculated by computing the ratio of the length, $L$ (the distance along the boat covered by the centerboard) to the height, $D$ (the amount the centerboard protrudes from the hull). This ratio is then used to find the value of $C$. For the purpose of this investigation, the ratio is 4.0 , which is a typical ratio for the centerboard of a collegiate FJ. This ratio results in a $C$ value of 1.12 . Thus, $F_{c b w}(7)$ is given by

$$
F_{c b w}=-0.56 \rho A_{c b} v^{2}
$$

The area of the centerboard of a collegiate FJ is about 0.4 square meters. Therefore, 
$F_{c b w}(8)$ is further simplified to

$$
F_{c b w}=-0.56 \rho(0.4) v^{2}=-0.224 \rho v^{2}
$$

To calculate the net force in the $x$-direction and the net force in the $y$-direction, vector addition must be used. The net force in the $x$-direction, denoted $F_{t x}$, is given by

$$
F_{t x}=F_{w x}+F_{r}
$$

Using in the formulas previously discussed for $F_{w x}$ (3) and $F_{r}(6)$ yields

$$
F_{t x}=-\frac{1}{\sqrt{2}} \frac{\Delta P M}{\rho_{\text {air }} d}+\mu_{R} A v^{2}
$$

The net force in the $y$-direction, denoted $F_{t y}$, is given by (4) plus (9)

$$
F_{t y}=F_{w y}+F_{c b w}
$$

which can be written

$$
F_{t y}=\frac{1}{\sqrt{2}} \frac{\Delta P M}{\rho_{\text {air }}}-0.56 \rho A_{c b} v^{2}
$$

To find the total net force (denoted $F_{t}$ ), the components (10) and (11) must be combined.

$$
F_{t}=\sqrt{{F_{t x}}^{2}+F_{t y}^{2}}=\sqrt{\left(-\frac{1}{\sqrt{2}} \frac{\Delta P M}{\rho_{\text {air }}}+\mu_{R} A v^{2}\right)^{2}+\left(\frac{1}{\sqrt{2}} \frac{\Delta P M}{\rho_{\text {air }}}-0.56 \rho A_{c b} v^{2}\right)^{2}}
$$

The density of air is known to be 


$$
\rho_{\text {air }}=1.20 \mathrm{~kg} / \mathrm{m}^{3}
$$

and the sailboat is assumed to be sailing in freshwater with a density of

$$
\rho_{\text {freshwater }}=10^{3} \mathrm{~kg} / \mathrm{m}^{3}
$$

The values (13) and (14) are applied to equation (12) yielding

$$
\begin{aligned}
F_{t} & =\sqrt{\left(-\frac{1}{\sqrt{2}} \frac{\Delta P M}{1.2 d}+\mu_{R} A v^{2}\right)^{2}+\left(\frac{1}{\sqrt{2}} \frac{\Delta P M}{\rho_{\text {air }}}-0.56\left(10^{3}\right) A_{c b} v^{2}\right)^{2}} \\
& =\sqrt{\frac{(\Delta P)^{2} M^{2}}{2.88 d^{2}}-\frac{\sqrt{2}(\Delta P) M}{1.2 d} \mu_{R} A v^{2}+\mu_{R}^{2} \mathrm{~A}^{2} \mathrm{v}^{4}+\frac{(\Delta P)^{2} M^{2}}{2.88 d^{2}}-560 \sqrt{2} \mathrm{~A}_{c b} v^{2}+313600 \mathrm{~A}_{c b}{ }^{2} v^{4}}
\end{aligned}
$$

Using the equation

$$
R=\frac{L v}{\frac{\mu}{\rho}}=\frac{L v \rho}{\mu}
$$

and the constants

$$
\begin{aligned}
\mu & =0.00000115 \\
\rho & =10^{3} \mathrm{~kg} / \mathrm{m}^{3} \\
L & =4.00 \mathrm{~m},
\end{aligned}
$$

where $L$ is the length of a collegiate FJ, the Reynolds number, $R$, can be found:

$$
R=\frac{4.00 v(0.001)}{0.00000115}=3478260870 v .
$$

Also 


$$
\mu_{R}=\frac{64}{3478260870 v}=\frac{1.84 \cdot 10^{-8}}{v}
$$

Substituting $\mu_{R}$ (16) into equation (15) yields

$F_{t}=$

$\sqrt{\frac{\Delta P^{2} M^{2}}{2.88 d^{2}}-\frac{\sqrt{2} \Delta P M}{1.2 d} 1.84 A v h+3.3856 A^{2} v^{3} h^{2}+\frac{\Delta P^{2} M^{2}}{2.88 d^{2}}-560 \sqrt{2} A_{c b} v^{2}+313600 A_{c b}{ }^{2} v^{4}}$

where $h=10^{-8}$.

The wetted surface area of the sailboat, $A$, is assumed to be $4.80 \mathrm{~m}^{2}$ and the surface area of the centerboard, $A_{c b}$, is $0.4 \mathrm{~m}^{2}$. Equation (17) becomes

$$
F_{t}=\sqrt{\frac{\Delta P^{2} M^{2}}{2.88 d^{2}}-\frac{\sqrt{2} \Delta P M}{1.2 d} 8.832 v h+78 v^{3} h^{2}+\frac{\Delta P^{2} M^{2}}{2.88 d^{2}}-224 \sqrt{2} v^{2}+50176 v^{4}}
$$

where $h=10^{-8}$ and 78 is used for the more accurate 78.004224 .

Based on a wind speed of around 4 knots, $\Delta P$ is approximately $500 \mathrm{~N} / \mathrm{m}^{2}$. The force (18) is then

$$
F_{t}=\sqrt{\frac{500^{2} M^{2}}{2.88 d^{2}}-\frac{500 \sqrt{2} M}{1.2 d} 8.832 v h+78 \mathrm{v}^{3} h^{2}+\frac{500^{2} M^{2}}{2.88 d^{2}}-224 \sqrt{2} \mathrm{v}^{2}+50176 \mathrm{v}^{4}}
$$

where $h=10^{-8}$. Assuming the sailboat has a velocity, $v$ of about 2 meters per second, a $d$ of about 9 meters (as the approximate distance covered by the sails), and a mass, $M$, of the sailboat and sailors of $250 \mathrm{~kg}$, (19) becomes 


$$
\begin{aligned}
F_{t} & =\sqrt{\frac{500^{2} 250^{2}}{2.88(9)^{2}}-\frac{125000 \sqrt{2}}{1.2(9)} 17.664 \cdot 10^{-8}+624 \cdot 10^{-16}+\frac{500^{2} 250^{2}}{2.88(9)^{2}}-896 \sqrt{2}+802816} \\
& =11609 \mathrm{~N}
\end{aligned}
$$

It is important to note that this total force has both an $x$-component and a $y$-component. This is significant because it means that the sailboat does not travel in a straight line relative to its bow because the $y$-component of the force pushes it sideways and the $x$-component of the force pushes it forwards.

\section{DISCUSSION}

The calculations in this project were made based on a collegiate FJ sailboat and, as discussed previously, a few necessary assumptions were made. However, the principles investigated here are the same for any sailboat, and the same basic calculations can be used to determine and analyze the forces acting on any sailboat.

In (20) it is found that the total force acting on the sailboat in about 4 knots of wind is $11609 \mathrm{~N}$. Therefore, given that the combined weight of the collegiate FJ and crew is approximately $250 \mathrm{~kg}$ and

$$
a=\frac{F}{M}=\frac{11609}{250} \approx 46.4 \mathrm{~m} / \mathrm{s}
$$

This acceleration (21) is the initial acceleration and is not constant, but rather it gradually decreases over time and eventually becomes zero as the boat reaches a constant velocity. Since 
this initial acceleration is reasonable, it helps to affirm that the calculations of the force acting on the sailboat are reasonable.

It is also important to note that torques are present in the sailboat system. There is a torque caused by the force of the sail/wind on the mast, a torque caused by the sailors sitting on the edge of the boat, and a torque caused by the centerboard/water interactions discussed previously. The calculation of these torques is beyond the scope of this project as the forces affecting a sailboat can be calculated and understood without complicating matters further by including the torque calculations.

\section{CONCLUSION AND RECOMMENDATIONS}

Through the analysis of the forces affecting a sailboat and the interaction between these forces, a greater understanding of the components of the net force on a sailboat is obtained. The role that the centerboard plays in preventing excessive sideways slippage is realized and evaluated resulting in a broadened understanding of the nature of a sailboat's motion. Perhaps the most important point obtained from this research is that sailboats do not travel in a straight line relative to their bow, but rather experience sideways slippage that is not entirely prevented by the centerboard. In addition to this, a method has been developed for calculating the total force on a sailboat. This method can be used to calculate the total force on any sailboat, in any wind speed, with any crew and can thus become a valuable tool for sailors and boat designers alike as it provides a mathematical rationale for techniques and tactics that are already used in competitive sailing. 


\section{NOMENCLATURE}

\begin{tabular}{|c|c|c|}
\hline Symbol & Description & Value \\
\hline$F_{m g}$ & Force of gravity & $\mathrm{N}$ \\
\hline$g$ & Acceleration due to gravity $\left(9.8 \mathrm{~m} / \mathrm{s}^{2}\right)$ & $\mathrm{m} / \mathrm{s}^{2}$ \\
\hline$M$ & Combined mass of sailboat and sailors & $\mathrm{kg}$ \\
\hline$F_{b}$ & Buoyant force & $\mathrm{N}$ \\
\hline$\rho_{\text {water }}$ & Density of water & $\mathrm{kg} / \mathrm{m}^{3}$ \\
\hline$V$ & Volume of water displaced by boat & $\mathrm{m}^{3}$ \\
\hline$F_{w}$ & Force of the wind & $\mathrm{N}$ \\
\hline$F_{w x}$ & $x$-component of $F_{w}$ & $\mathrm{~N}$ \\
\hline$F_{y x}$ & $y$-component of $F_{w}$ & $\mathrm{~N}$ \\
\hline$\Delta P$ & Change in pressure & $\mathrm{Pa}$ \\
\hline$\rho_{\text {air }}$ & Density of air & $\mathrm{kg} / \mathrm{m}^{3}$ \\
\hline$F_{r}$ & Resistance force & $\mathrm{N}$ \\
\hline$R$ & Reynolds Number & dimensionless \\
\hline$L$ & Length of boat & $\mathrm{m}$ \\
\hline$v$ & Velocity & $\mathrm{m} / \mathrm{s}$ \\
\hline$\mu_{R}$ & Coefficient of friction & dimensionless \\
\hline$A$ & Area of hull underwater & $\mathrm{m}^{2}$ \\
\hline$F_{c b w}$ & Force resulting from centerboard-water interactions & $\mathrm{N}$ \\
\hline$C$ & Drag coefficient & dimensionless \\
\hline$A_{c b}$ & Area of centerboard & $\mathrm{m}^{2}$ \\
\hline$F_{t x}$ & Net force in $x$-direction & $\mathrm{N}$ \\
\hline$F_{t y}$ & Net force in $y$-direction & $\mathrm{N}$ \\
\hline$F_{t}$ & Total net force & $\mathrm{N}$ \\
\hline$a$ & Acceleration & $\mathrm{m} / \mathrm{s}^{2}$ \\
\hline
\end{tabular}




\section{APPENDIX \\ NAUTICAL TERMS}

Bow - the front of the boat.

Centerboard - a board that protrudes out of the bottom of the boat and helps prevent the boat from simply slipping sideways.

Hull - the boat excluding the sails, mast, and centerboard.

$\mathrm{Jib}$ - the smaller of the two sails, located towards the bow.

Leeward - the side of the boat farther downwind (in this case, the starboard side).

Mainsail - the bigger of the two sails, located towards the stern.

Mast - the metal pole that holds the sails up.

Port Side - the left side of the boat when looking at the boat from the stern.

Shroud - a wire that runs from the hull to the mast (port and starboard shroud).

Starboard Side - the right side of the boat when looking at the boat from the stern.

Stern - the back of the boat.

Windward - the side of the boat farther upwind (in this project, the port side).

\section{REFERENCES}

"Collegiate FJ." FJ Sailboat - Sailboats for Sale. Zim Sailing, 2015. Web. 15 Nov. 2015. <http://www.zimsailing.com/collegiate-fj.html>.

"Drag Force, Velocity, and Area Calculation." LMNO Engineering, Research, and Software.

LMNO Engineering, Research, and Software, Ltd, 2008. Web. 26 Nov. 2015. 
<http://www.lmnoeng.com/Force/DragForce.php>.

"Friction Coefficient at Laminar Flow." The Engineering Toolbox. The Engineering Toolbox.

Web. 22 Nov. 2015.

<http://www.engineeringtoolbox.com/laminar-friction-coefficient-d_1032.html>.

Garrett, Ross. The Symmetry of Sailing: The Physics of Sailing for Yachtsmen. Dobbs Ferry:

Sheridan House, 1996. 209-212. Print.

"Lateen Sail." Encyclopedia Britannica Online. Encyclopedia Britannica, 2015. Web. 13 Nov. 2015. <http://www.britannica.com/technology/lateen-sail>.

"Liquids - Kinematic Viscosities." The Engineering Toolbox. The Engineering Toolbox. Web. 17

Nov. 2015. <http://www.engineeringtoolbox.com/kinematic-viscosity-d_397.html>.

"Suomi Virtual Museum." Pressure Gradient Force. Verner E. Suomi Virtual Museum, 1999.

Web. 22 Nov. 2015.

<http://profhorn.meteor.wisc.edu/wxwise/museum/a10/a10pressure.html>

Waters, Mike. "Boat Resistance." Small Trimaran Design. Mike Waters, 2010. Web. 24 Nov.

2015. <http://www.smalltridesign.com/Trimaran-Articles/Boat-Resistance.html>. 